The team numerically investigated the Lorentz forces acting on a free electron gas that was bound to the surface of a gold nanowire under linearly-polarized electromagnetic plane waves in the wavelength range from $400 \mathrm{~nm}$ to $1,200 \mathrm{~nm}$. The diameter and length of the nanowire was $75 \mathrm{~nm}$ and $1,025 \mathrm{~nm}$, respectively. They found that plasmonically-induced Lorentz forces were significant and stronger than electric-dipole induced forces, particularly for the case of long-wavelength excitation between plasmon resonances. The existence of chiral plasmons coincided with spiral magnetic field patterns that produced net transverse forces and torques. Theoretical models that consider only electric-dipole induced torques cannot explain the experimental results - in particular, they fail to explain why the nanowires align perpendicular to the linear polarization of an optical trap. However, the model proposed by the researchers can convincingly explain it.

\section{SENSORS \\ Nanoscale magnetometer} Nano Lett. 14, 6443-6448 (2014)

Developing a nanoscale magnetic sensing technique applicable to individual molecules could enable revolutionary advances in the physical, biological and medical sciences. However, such magnetic sensing devices ideally require the ability to perform sensitive detection of very weak magnetic fields with nanometre-scale resolution in ambient conditions at room temperature. Now, Alex Sushkov and co-workers from the USA China, Germany and Russia have developed an all-optical method to detect the electron spin of a single molecule using a shallow nitrogen-vacancy (NV) centre in bulk diamond. The proof-of-principle experiment was implemented by attaching paramagnetic molecules containing $\mathrm{Gd}^{3+}$ to the surface of a bulk diamond crystal with widely separated $\mathrm{NV}$ centres located at a nominal depth of $6 \mathrm{~nm}$ below the diamond surface. With the aid of gold nanoparticles as markers, the positions of the NV centres and the molecules were determined by fluorescence imaging and atomic force microscopy. The international team of researchers measured the spin-statedependent fluorescence following a $532 \mathrm{~nm}$ laser pulse with variable delay time. When the NV centre was in the proximity of a $\mathrm{Gd}^{3+}$ molecule, the measured NV spin-state population relaxation rate was significantly increased. Once the molecule was removed, the relaxation returned to the intrinsic rate. The sensing radius of an NV centre was determined to be approximately $12 \mathrm{~nm}$.

\section{PLASMONICS}

\section{Electromagnetic wormholes} Phys. Rev. A 90, 043812 (2014)

Transformational plasmonics has recently emerged as a research topic to describe and exploit the coupling between surface electrons and incident light. However, an analogy with general relativity has also led research in another direction. Now, Muamer Kadic and co-workers from the Fresnel Institute in Marseille, France report using the plasmonic analogues of electromagnetic wormholes for controlling the propagation of surface plasmon polaritons. They focus on two different designs that fit the purpose: a toroidal cloak - a so-called handlebody that connects two holes on a metal surface - and the same toroidal cloak lying flat on a metal surface, resembling a doughnut. In both cases, full-wave numerical simulations show that surface plasmon polaritons propagate undisturbed in the metal, independent of the presence of an electromagnetic field in these metastructures. The computations, based on the finite element method, make the leap from the two- to the threedimensional case, taking into account the plasmon polarization as well as the permittivity change at the interface between the different materials and air. Hoping to inspire experimental demonstrations of their results, the authors also infer the multilayered versions of the proposed wormhole analogues for applications within the visible range. Simulations reveal unperturbated propagation of the wavefronts from a plasmon source in the middle for both the handlebody and doughnut cases. To create an actual device, you need homogeneous isotropic magnetic layers for the handlebody design and homogeneous isotropic dielectric layers for the doughnut design.

$M M$

\section{BLACK HOLES}

\section{On the lab table}

Nature Phys. 10, 864-869 (2014)

Stephen Hawking's name has been associated with black holes - regions of space and time from which no light or matter within the so-called event horizon can escape. Although Hawking predicted in 1974 that black holes emit radiation, astronomers have not been able to observe it as it is expected to be incredibly weak. Now, Jeff Steinhauer from Technion-Israel Institute of Technology in Haifa reports the realization of an analogue event horizon in the lab using the transition from subsonic to supersonic flow in a Bose-Einstein condensate. The flow of the condensate was accelerated to velocities $\mathrm{NH}$ that exceed the speed of sound, trapping sound waves in this supersonic region, just as gravity traps anything within a black hole. Steinhauer observed the appearance and disappearance of pairs of sound waves on this border, with a small part forming across the horizon and resembling Hawking radiation. A secondary potential within the first was used to further trap and amplify this Hawking radiation in a laser-like operation, so that it could be detected experimentally. This result not only provides a way of studying black-hole physics in a laboratory environment but may also prove useful for studying the link between quantum theory and gravity, as Hawking radiation relies on both.

\section{GRAPHENE}

\section{Fibre integration}

Optica 1, 307-310 (2014)

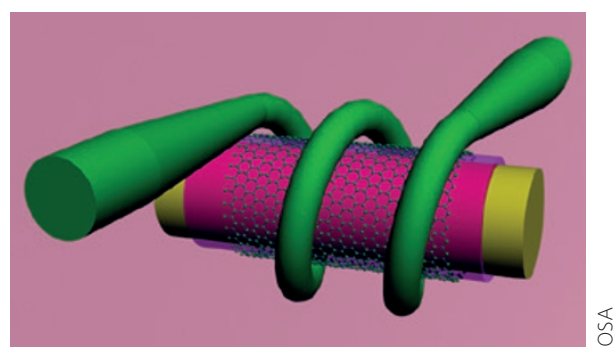

Wrapping optical microfibre coils around a polymer rod coated with a graphene sheet can yield compact polarizers and high- $Q$ resonators, report scientists from China. Jun-Long Kou and co-workers from Nanjing University say that the approach provides an enhanced and long interaction path between the evanescent light field of the microfibre and the graphene. This interaction can then be exploited for applications such as polarization manipulation or forming a single-polarization resonator. To prevent optical loss from the high-index polymer rod (a piece of PMMA with a diameter of $2 \mathrm{~mm}$ ) a thin layer of Teflon is applied to the rod's surface prior to application of the graphene sheet, which is made by chemical vapour deposition and then mechanically transferred. Preliminary experiments suggest that a polarizer based on the approach can offer an extinction ratio of $8 \mathrm{~dB}$ per each microfibre coil, with a bandwidth of $450 \mathrm{~nm}$ in the $1.5 \mu \mathrm{m}$ telecommunications window. Possibilities for future research include attempting the approach with other two-dimensional materials instead of graphene, such as the transition metal dichalcogenides.

Written by Oliver Graydon, Noriaki Horiuchi and Maria Maragkou. 